\title{
The Design of Rapid Thermal Process for Large Diameter Applications
}

\author{
C. W. Liu*, M. H. Lee, C. Y. Chao, C. Y. Chen, and C. C. Yang \\ Department of Electrical Engineering and, National Taiwan University, Taipei, Taiwan \\ Y. Chang, \\ Dept. of Mechanical Engineering, Chung Cheng Inst. of Technology, Taoyuan, Taiwan \\ *email : chee@cc.ee.ntu.edu.tw
}

\begin{abstract}
In this paper, we will address issues of the power consumption of the lamps, temperature uniformity across wafer, and the grating temperature measurements, for large diameter wafer process. The optimum design of the lamp separation and the distance between the lamp arrays and wafer surface can be obtained with the uniform radiation and the minimum lamp power. Based on a simplified radiation thermal model, the transient and steady state non-uniformity of temperature on the wafer edge can be predicted. The wafer emissivity as a function of temperature and wavelength is exactly modeled. The effect of multi-layer grown on $\mathrm{Si}$ was also included. A grating temperature measurement was demonstrated in RTP process. We used the laser ablation technique to fabricate $\mathrm{Si}$ grating. To increase the sensitivity of measurements, a large angle diffracted beam was used.
\end{abstract}

\section{Introduction}

Due to the demand of single wafer process for the manufacture of large diameter wafers, which reduces the factory costs and production cycle time, the interest of rapid thermal process (RTP) continues to grow with advantages of reduced thermal budget and cold wall system. The trend to larger wafer sizes has been related to enhanced process control to guarantee homogenous conditions across the wafer. The uniformity of the temperature distribution across the wafer depends on the irradiation distribution of the lamp arrays. Consequently, the temperature distribution on the wafer can be influenced by lamp arrangement, the radiation power of each lamp, the size and optical properties of the wafer.

Temperature measurement is also key issue in RTP. The methods to measuring temperature are contact and non-contact techniques. Pyrometry is the most used noncontact technique for temperature measurement in RTP chamber and it dependence on emissivity of Si. Therefore, the real wafer temperature could be obtained with a completely 
modeling emissivity. However, the emissivity depend on temperature, doping, layer structures, surface patterns and back side roughness, causes some severe temperature measurement error, especially at low temperature $\left(<600^{\circ} \mathrm{C}\right)$. Nevertheless, the thermal expansion coefficient of $\mathrm{Si}$ is relatively independent on process variation, and is between 2 to $5 \times 10^{-6} \mathrm{~K}^{-1}$ from room temperature to $1000^{\circ} \mathrm{C}$. The relative constant of thermal expansion coefficient makes it attractive to measure the Si wafer temperature. Therefore, we investigated the grating prepared by the ablation of high power laser interference, and used these gratings to measure the Si wafer temperature by monitoring the thermal expansion of grating periods. The grating temperature measurement is applicable to other material system. Similar gratings were also fabricated on $\mathrm{GaAs}, \mathrm{InP}, \mathrm{GaN}$ and $\mathrm{LiNbO}_{3}$ for the temperature measurement.

\section{Irradiation Intensity}

The distribution of intensity of the wafer under tubular lamps irradiation like the combination of infinite point-like source which emits its radiation equally in all directions [1]. Because there is a reflective-plane-like a mirror above the tubular lamps, so it also. adds the reflection effect. Due to the thermal radiation, the wafer itself also emits light. The virtual image of the wafer therefore illuminates the actual wafer creating a second feedback [2]. The third and more high order feedback are too smaller than the second one and thus can be neglected. But the light reflected by the mirror would be screened a part of energy by tubular lamps. Therefore, the reflected quantity must be multiplied a screening factor. The screening factor can be measured by experiment. If there is no tubular lamps, the value of the factor should be 1 , because the reflected quantity is very small.

We consider that a set of the tungsten-halogen lamp tubes are all length $30 \mathrm{~cm}$, with separation of $\Delta S=2 \mathrm{~cm}$ from each other and the power of $2 \mathrm{KW}$ for the single lamp, and there are 18 lamp tubes which are arranged in a parallel direction and at the same plane. But the efficient power of a single lamp just have $50 \%$. Fig. 1 shows the simulation results which are the intensity profiles along the middle of lamp tubes with different distance $h$ between the lamps and the wafer, without the reflection effect. From Fig.1, we can know the intensity distribution is depending on $h / \Delta S$ ratio. Fig. 2 shows the relation between ripple and different $h$, when ripple is define as (max/min)- 1 of the intensity profile. For the ratio smaller than 0.6 , there are more than $10 \%$ intensity variation, which will cause the temperature non-uniformity across the wafer. On the other hand, if we increase $h$, the ratio will be small with the disadvantage of power consumption. Fig. 3 shows the total absorbed power of 8 " wafer with different $h$. So the optimum ratio should be nearly about 1, i.e. $h$ about equal to $\Delta S$. Fig. 4 shows the total intensity profile with reflection effect when $\Delta S=h=2 \mathrm{~cm}$ and the distance between the mirror and lamps plane is also $2 \mathrm{~cm}$. Note that the 
effect of chamber geometry was neglected, and the heat flux from the lamps was assumed to uniform over the wafer. Similar results have been report in Ref.1 and Ref.2. However the lamp element model in Ref.2 is incorrect, since the energy is not conserved in this model.

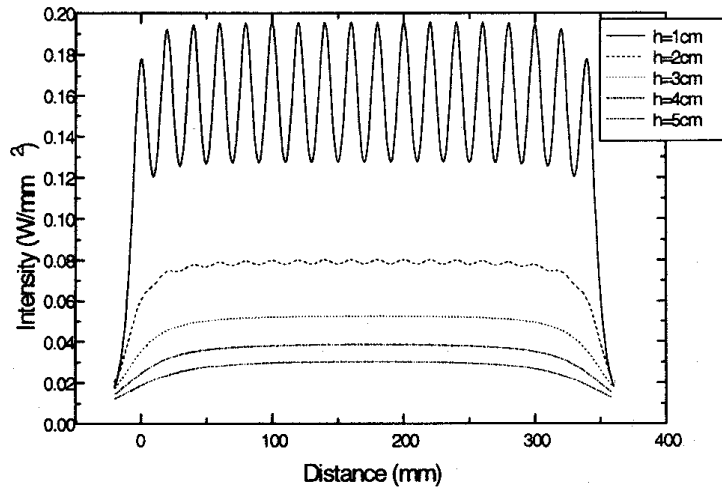

Fig. 1 The profile along the middle of lamp.

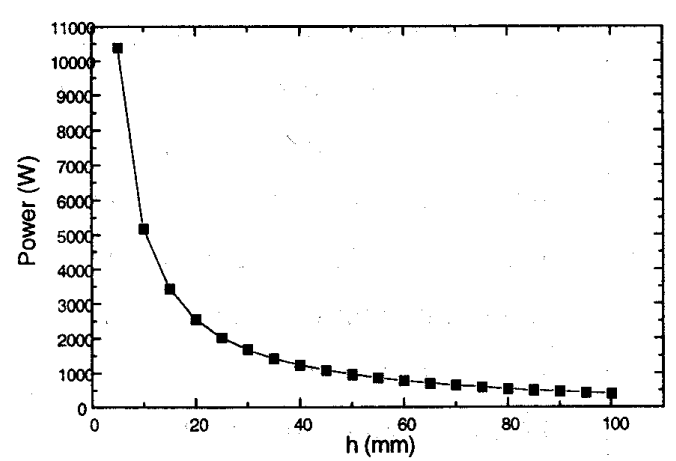

Fig. 3 The total power with differential lamp height of illumination on 8 " wafer.

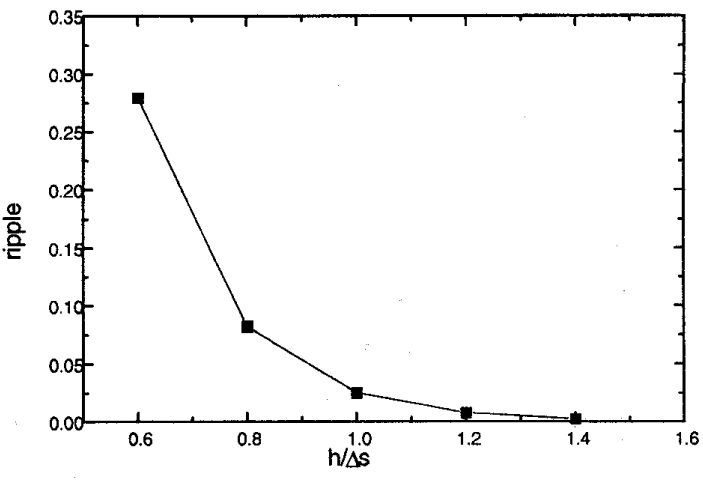

Fig.2 The ripple((max. of intensity/min. of intensity)-1) as a function of the ratio between $h$ and $\Delta S$.

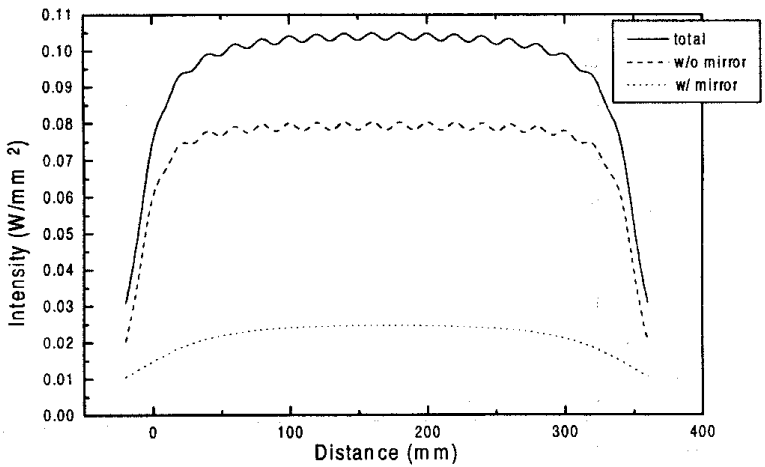

Fig.4 The profile of directional irradiation, reflected by mirror and total intensity

\section{Transient and Steady State edge Non-uniformity}

One of the main problems of RTP is the temperature decrease near the wafer edge. Because the wafer edge does not normally receive radiation from the lamps which results in a net radiative energy loss from the edge [3] at steady state. For the standard of $0.18 \mu \mathrm{m}$ technology, the temperature difference between the center and the edge of wafer must be smaller than $3^{\circ} \mathrm{C}$. Fig. 5 shows the relation between the edge non-uniformity and wafer size 
at steady state $800^{\circ} \mathrm{C}$. The edge width is the distance from edge to the radial position where the temperature variation is equal to $3^{\circ} \mathrm{C}$ as compared to the center. When wafer size increases the available area is relatively large. The thicker wafer can have better uniformity as shown in Fig.5. Fig.6 shows the edge non-uniformity of various wafer sizes at different steady state temperature, if a simple linear tube array is used. This indicates that simple linear lamp array can have better uniformity as the wafer size increases.

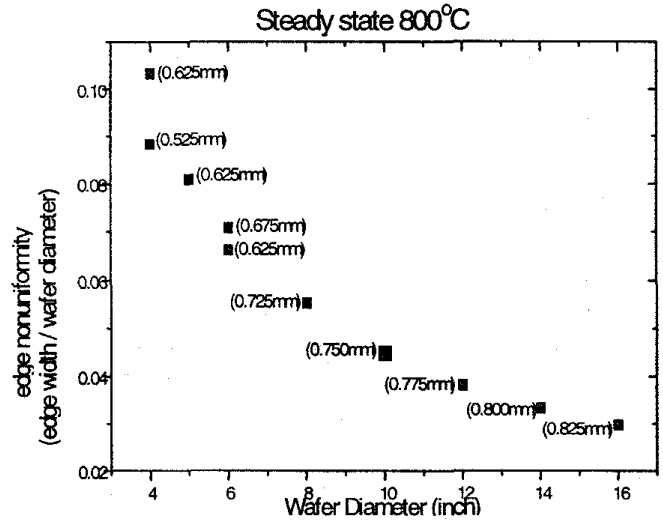

Fig.5 Edge non-uniformity = edge width $/$ wafer diameter. The brackets are the thickness of wafer.

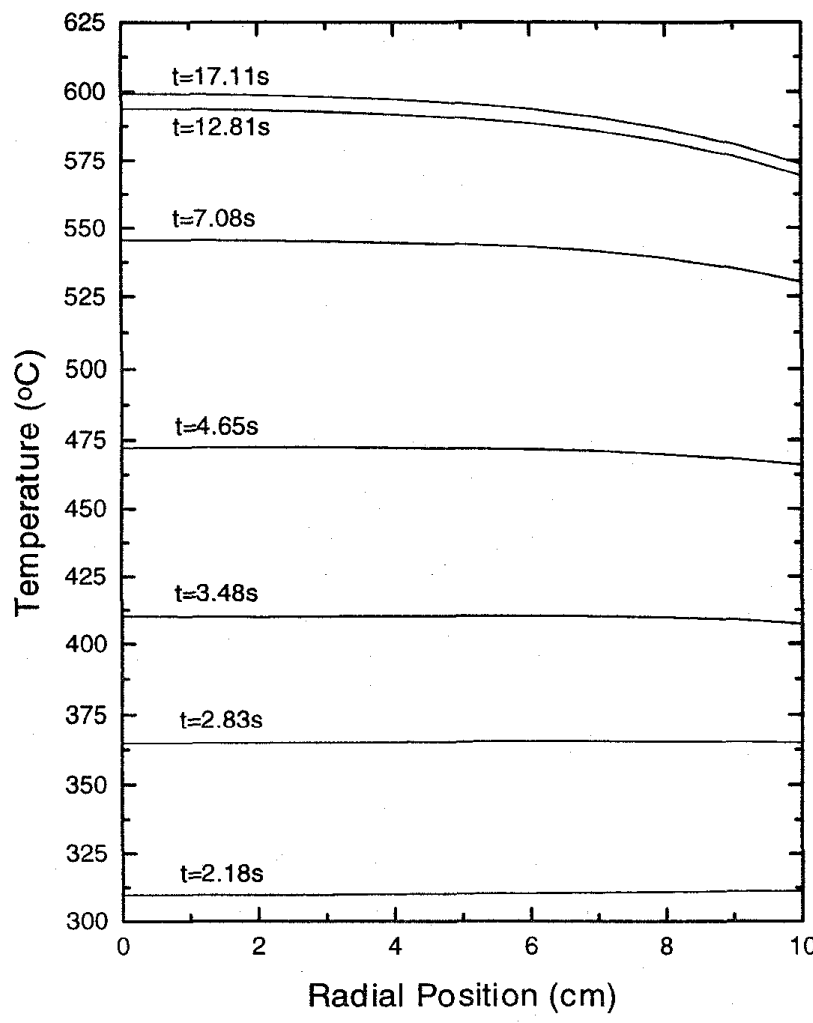

Fig. 7 The transient temperature profile at steady state $600^{\circ} \mathrm{C}$.

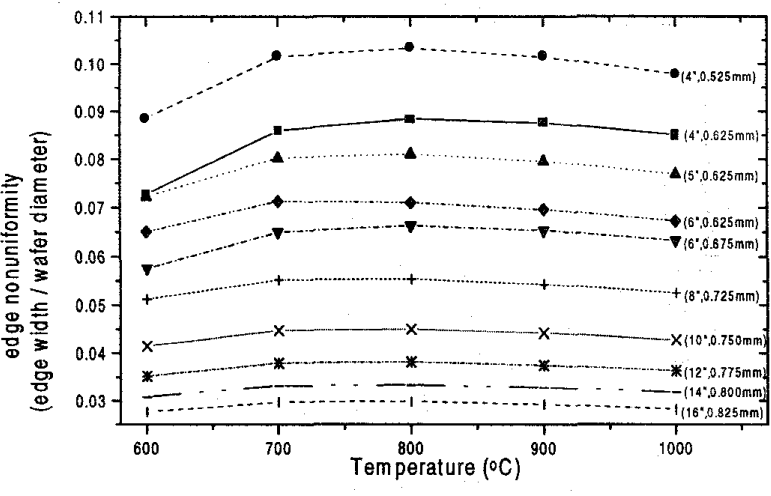

Fig. 6 The edge non-uniformity with various wafer sizes and temperatures.

For the transient analysis, the temperature of the edge is higher than center at first several seconds when ramp is up. Because on the front side only the heat flux which direction is perpendicular wafer can reach the side, at the edge not only perpendicular direction but also parallel direction that can heat the wafer. Fig. 7 shows the transient simulation, this phenomenon is still about 3 seconds. In RTP, the fast ramp up and down is usually smaller than half minute, so this phenomenon could be considered.

The calculation of the temperature distribution in the 
wafer is based on solving heat conduction equation, and it is usually non-linear. Because there is a term which energy flux flow into the wafer, it is proportional $T^{4}$, where $T$ is temperature. We develop a set of small signed model to make it linear, also can predict the transient lifetime $\tau$

$$
\frac{1}{\tau}=\frac{b+4 \cdot a \cdot T^{3}}{m}
$$

where $a$ is $7.938 \times 10^{-12} \mathrm{~W} / \mathrm{cm}^{2} \mathrm{~K}^{4}$ for $\mathrm{Si}, b$ is convective heat transfer coefficient and $m$ is also a constant but depend on wafer thickness, Si density and specific heat.

\section{Model-Based Emissivity Correction in Pyrometer Temperature}

Pyrometry temperature measurement is the most suitable for RTP system and most used in current the technology. It doesn't have the disadvantages of contamination as the thermocouple. But using pyrometry to measure temperature must have sufficient knowledge for the optical properties of $\mathrm{Si}$, especially the spectral emissivity. Fig. 8 shows the results to simulate the normal spectral emissivity of a lightly doped $700 \mu \mathrm{m}$ Si wafer with different temperature and wavelength. Actually, there are not only Si on the wafer, some material could be coated on it, like $\mathrm{SiO}_{2}$ and poly-Si. Therefore, the emissivity is different for multi-layer structure. The simulation results all also show in Fig.9. The emissivity looked like a little periodical. Because it is a function of poly thickness and can produce dramatic change in the deposited film as the optical path near 1/4 of the pyrometer wavelength [4]. Pyrometer temperature measurement is made using the radiation emitted at the center of a certain narrow wavelength band from the wafer surface. In Sorrell's paper [4], we can get the expression of pyrometer temperature $T_{p}$

$$
T_{p}=\frac{C_{2}}{\lambda \cdot \ln \left(1+\frac{\varepsilon_{p}}{\varepsilon_{w}}\left(e^{C_{2} / \lambda T_{w}}-1\right)\right)}
$$

where $T_{w}$ is the wafer temperature, $C_{I}$ and $C_{2}$ is $3.7418 \times 10^{8} \mathrm{~W} \cdot \mu \mathrm{m}^{4} \mathrm{~m}^{-2}$ and $1.4388 \times 10^{4} \mu \mathrm{m} \cdot \mathrm{K}, \lambda$ is the center wavelength of the narrow band, and $\varepsilon_{p}$ and $\varepsilon_{w}$ are the constant 0.7 and the actual surface emissivity. Therefore, with a constant $T_{w}$, we can predict pyrometer temperature $T_{p}$ as a function of poly thickness. In steady state, temperature control system of RTP make erroneous wafer temperature if constant emissivity is used. 


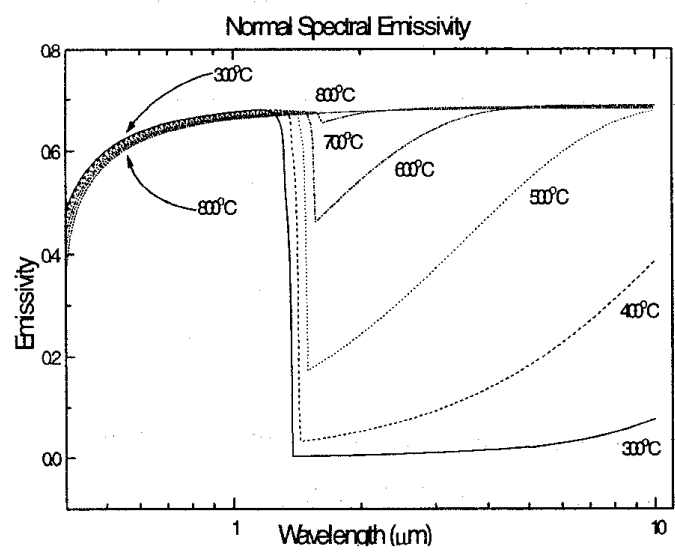

Fig. 8 The normal spectral emissivity of a lightly doped $700 \mu \mathrm{m}$ Si wafer.

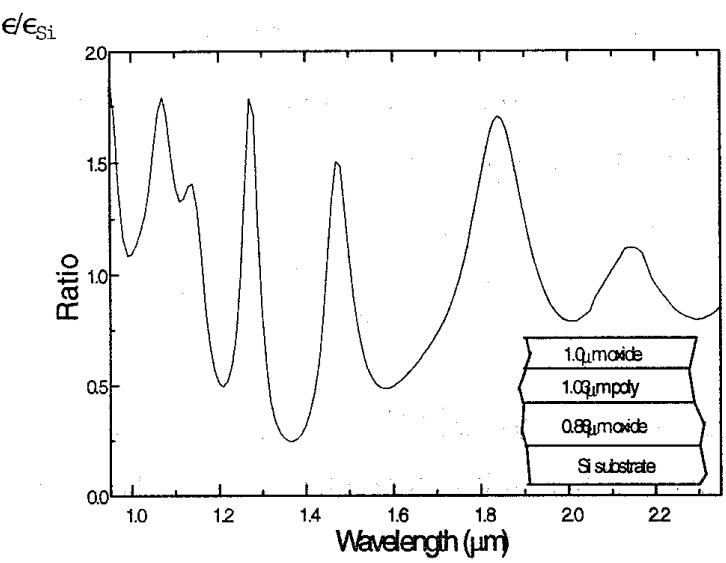

Fig.9 The ratio of the spectral emissivity of a multilayercoated Si sample to that of plain $\mathrm{Si}$, for the case of $45^{\circ}$ angle-of-incidence and the s-polarization. The thin film coating structure is shown in the insert.

\section{Roughness Dependent Emissivity}

In many RTP systems pyrometers monitor radiation emitted from the wafer rough backside, and there roughness effect can introduce additional errors in temperature measurement [5]. Fig. 10 shows the reflectivity on three kind of roughness surface at $300^{\circ} \mathrm{C}$ for different solid angle from $0^{\circ}$ to $30^{\circ}$ for pyrometer wavelength $1 \mu \mathrm{m}$ and $8 \mu \mathrm{m}$. $\sigma$ and $T$ are the standard deviation and lateral correlation length. Note the emissivity equal 1 reflectivity. The effect of surface roughness on the reflection qualitatively scale as $\sigma / T$. Clearly, as $\sigma / T$ increase as the surface becomes rougher, the reflectance saturates at a

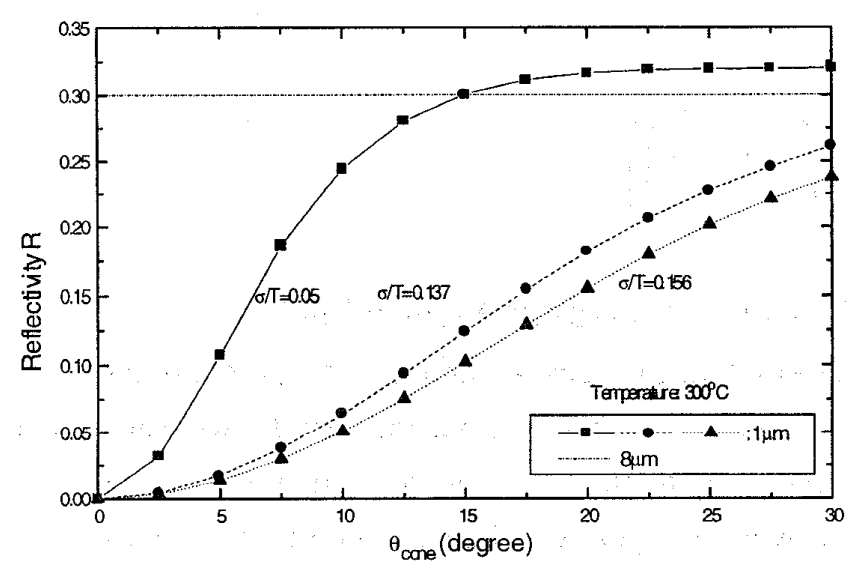

Fig. 10 The reflectivity on roughness surface at $300^{\circ} \mathrm{C}$ for different solid angle. $\sigma$ and $T$ are the standard deviation and lateral correlation length. bigger $\theta_{\text {cone }}$. The wafer surface of sample \#1, \#2 and \#3 should be smooth for light of $\lambda=8 \mu \mathrm{m}$. The reflective at $8 \mu \mathrm{m}$ is similar to perfectly smooth Si surface. Therefore, we chose wavelength of $8 \mu \mathrm{m}$ for our pyrometry. Besides, it also can avoid the interference from the lamp.

\section{Grating Fabrication}

The Si gratings were ablated by the interference of ultraviolet 
laser beams with the wavelength of $266 \mathrm{~nm}$, which is the fourth harmonic of a Q-switched Nd:Yag laser. The setup is shown in Fig.11. The laser beam of $2 \times 0.5 \mathrm{~cm}^{2}$ can be formed after the refraction of the two cylindrical lens, and then transmitted through fused silica prism. The prism is designed such that part of the laser beam is totally reflected on one edge, and the reflected beam with the other part of the laser beam transmitted through the other edge of the prism, where the Si sample is placed. There is an air gap between the $\mathrm{Si}$ surface and the prism edge to control the corrugation depth of the gratings. A large gap yields small grating depth. With high power level, the Si surface is melted and oxidized. Due to the interference of the two laser beams, the periodical corrugations can be formed. The grating period is controlled by the relative position of the prism, and can be varied from 180 to $550 \mathrm{~nm}$. The details of the grating fabrication can be found in Ref.6.

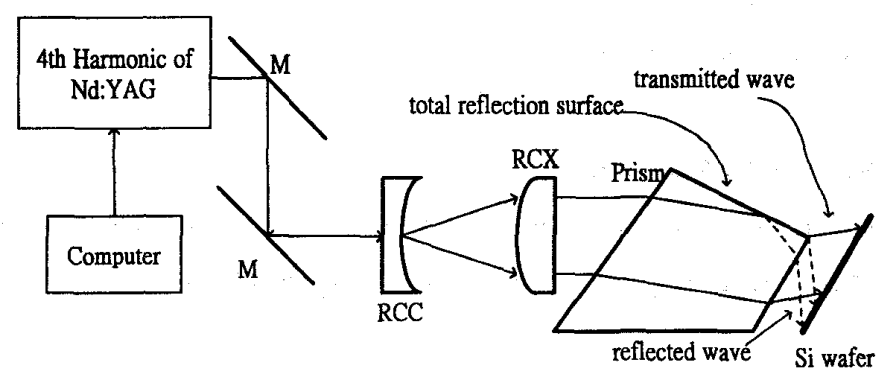

Fig.11 Schematic diagram of the grating fabrication on Si wafer using laser beam interference.

The composition of the $\mathrm{Si}$ grating is studied by Fourier transform infrared spectroscopy(FTIR) and energy dispersive $\mathrm{x}$-ray spectroscopy(EDX). The Si sample irradiated with the laser beam without any prism (no interference) is used for the FTIR measurement. Beside the normal oxide peak around $1100 \mathrm{~cm}^{-1}$, there is an extra shoulder in the FTIR spectra as compared to thermal oxide (Fig.12). This indicates the oxygen is deficient in the laser induced oxide [7]. The oxygen comes from the water vapor in the air, since the oxide fabricated in pure oxygen environments is much thinner than in the air. For some other experiments conducted in our lab, we found the moisture in the air can change the relative intensity between the shoulder and the main peak. To study the composition difference between the peak and valley, the EDX with $4.5 \mathrm{KeV}$ electron (penetration depth $<1 \mu \mathrm{m}$ ) and $50 \mathrm{~nm}$ spot size is used to probe the grating. It is observed that the oxygen signal in peak is stronger than that in valley, indicating the oxide in the peak is thicker than that in the valley. This is plausible, since the constructive interference can melt more $\mathrm{Si}$ and thus more oxide is formed after the incorporation of water vapor, as compared to the destructive interference. Also note that the intensity of the reflected beam and transmitted beam is not exact the same, because the destructive interference is not zero and is able to melt the Si surface. 


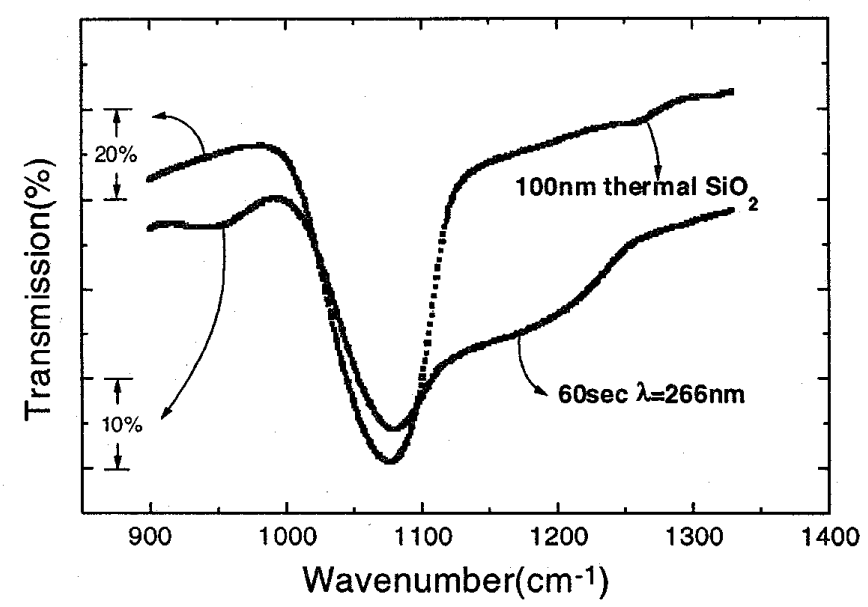

Fig.12 FTIR spectra of the laser induced oxide and the thermal oxide. The extra shoulder in the laser induced oxide indicates the $\mathrm{O}$ deficiency in the oxide films.

\section{Grating Temperature Measurement}

A reflection method is used to monitor the grating periods at different temperature. The -1 order of reflection beam is governed by the grating equation:

$$
\sin \theta_{-1}=\sin \theta_{i}-\frac{\lambda}{\Lambda}
$$

where $\lambda$ is the wavelength of incident beam, $\theta_{i}$ is the incident angle, and $\theta_{-1}$ is the reflecting angle. In practice, angular position of the normal reflection beam $\left(0^{\text {th }}\right.$ order $)$ has to be also measured to find the normal direction of the Si surface. The HeNe laser $(\lambda=633 \mathrm{~nm})$ was used as the incident beam, and the grating period is chosen as about $480 \mathrm{~nm}$. From the grating equation, the $\lambda / \Lambda$ ratio has to be less than 2 , but the large ratio can increase the sensitivity. The sensitivity can be derived from grating equation as $d \theta / d T=\lambda \alpha /\left(\cos \theta_{-1}\right.$ $\Lambda$ ), where the $\alpha$ is the thermal expansion coefficient. To increase the sensitivity, the reflection angle of -1 order beam is biased around $75^{\circ}$, which can increase the sensitivity by a factor of 5 as compared to the refection beam emerging normal to the surface [8]. The experimental setup is shown in Fig.13. The wafer was heated on a hot plate from room temperature to $800^{\circ} \mathrm{C}$. The reflection angle was calibrated from the beam position on a ruler. To account for the tilt of the Si surface during the heating, the angle of the $0^{\text {th }}$ order reflection was also measured for each temperature measurement step. Fig.14 shows the measurement results. For both increasing temperature and decreasing temperature scans, the curves were quite similar and consistent with the theoretical curves (solid line) based on empirical data of thermal expansion coefficient [9]. The slight scattering of the measurement data is due to the vibration of the laser beams. The wafer is placed in the air, 


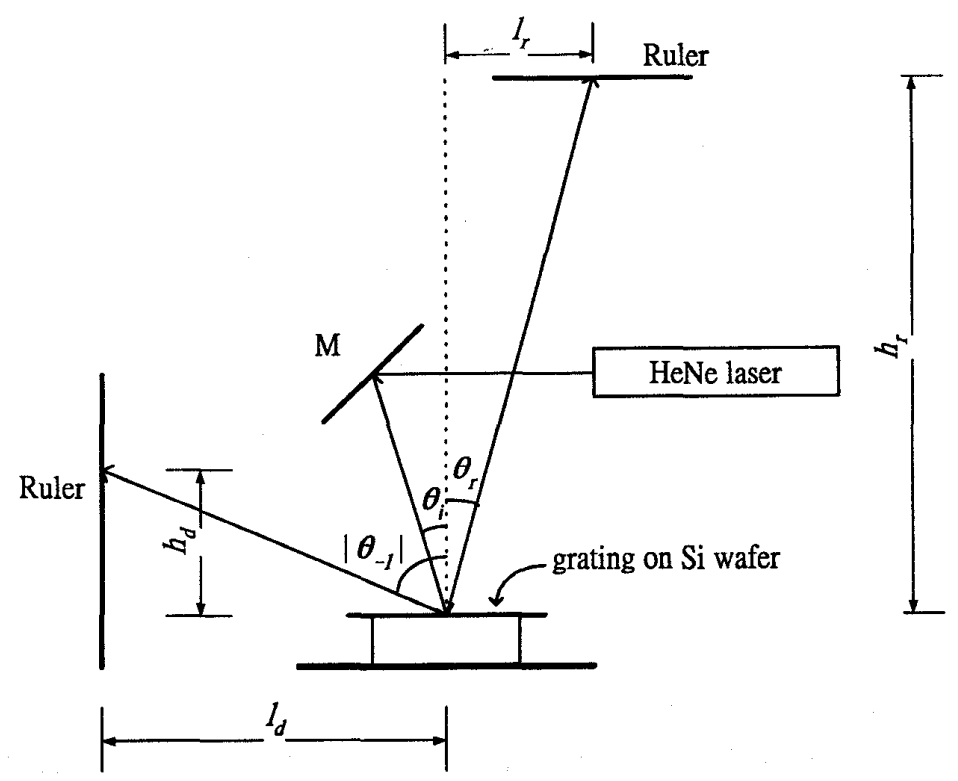

Fig.13 A schematic set-up of Si grating temperature measurement for the rapid thermal process.

the heating can cause the turbulence due to the thermal gradient. The effect will become

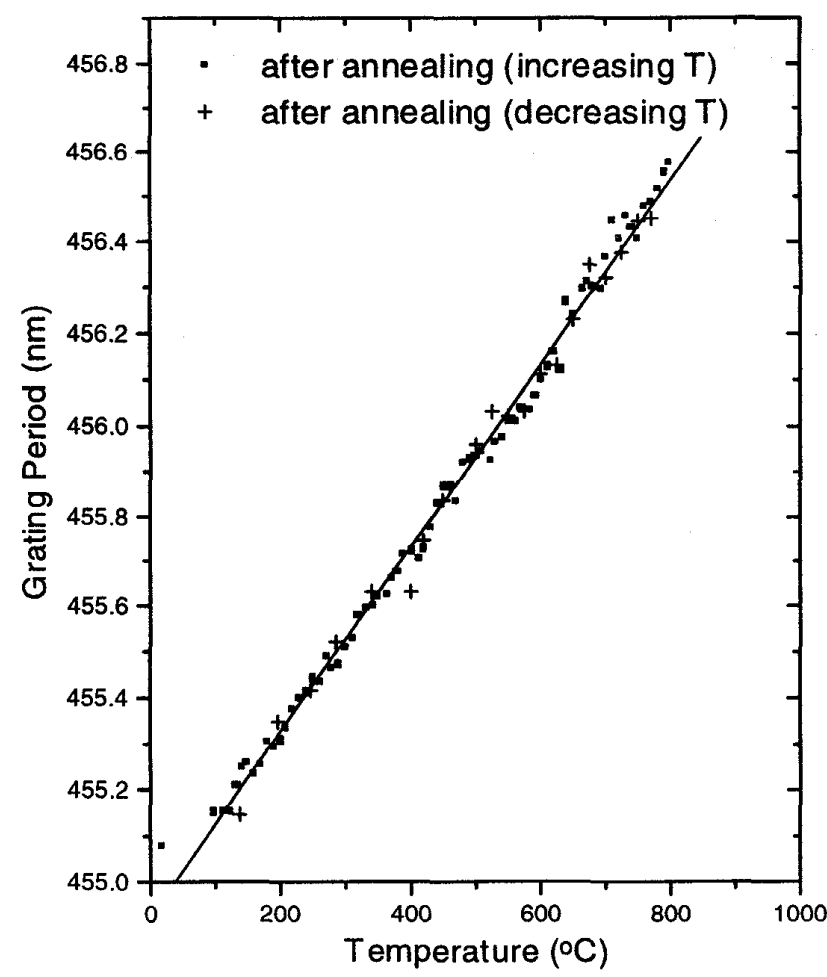

Fig. 14 Temperature measurement results for both increasing and decreasing temperature scans. small for the low pressure rapid thermal process, since the vibration magnitude is roughly proportional to the inverse of process pressure. For some gratings with thick oxide, the curve of measured period vs. temperature was irregular for the temperature less $400^{\circ} \mathrm{C}$. However, the period varied regularly after the rapid thermal annealing in nitrogen at $800^{\circ} \mathrm{C}$ for $30 \mathrm{sec}$. This effect may be attributed to the release of the thermal strain, which is built during the melting and fast quench on the $\mathrm{Si}$ surface in the laser ablation process. The effect can be avoided if the adequate exposure parameters are used in the fabrication of Si gratings. The temperature measurement from room temperature to $800^{\circ} \mathrm{C}$ was 
demonstrated.

\section{Summary and Acknowledgments}

We have studied the temperature uniformity and measurement of the wafer in RTP. The optimum design of temperature uniformity and power consumption can be obtained. The complete model of wafer emissivity is also obtained. In temperature measurement, using the thermal expansion of grating to measure the wafer is better and more accurate than convention method, and this work is supported by National Science Council (87-2221-E002-005, 86-2215-E-002-010).

\section{Reference}

[1] T. Theiler, Mat. Res. Soc. Symp. Proc. 387,55 (1995)

[2] J. M. Dilhac, N. Nolhier, C. Ganibal, C. Zanchi, IEEE Trans. Semiconduct. Manufact. vol. 8 , no. 4,432 (1995)

[3] R. Kakoschke, Mat. Res. Soc. Symp. Proc. 224,159 (1991)

[4] F. Y. Sorrel and R. S. Gyurcsik, IEEE Trans. Semiconduct. Manufact. vol. 6, no. 3, 273 (1993)

[5] H. Xu and J. C. Sturm, Mat. Res. Soc. Symp. Proc. 387,29 (1995)

[6] C. Y. Chao, C. Y. Chen, C. W. Liu, Y. Chang, and C. C. Yang, Appl. Phys. Lett. 71, 2442(1997).

[7] A. Strass, W. Hansch, F. Kaesen, A. Neubecker, P. Bieringer, A. Fisher, and I. Eisele, Technical Digest, the $7^{\text {th }}$ international symposium on silicon molecular beam epitaxy, Banff, Canada, 203(1997).

[8] S. H. Zaidi, S. R. J. Brueck, and J. R. McNeil, J. Vac. Sci. Technol. B 10, 166(1992). [9] Y. Okada and Y. Tokumaru, J. Appl. Phys. 56, 314(1884) 